BULLETIN (New Series) OF THE

AMERICAN MATHEMATICAL SOCIETY

Volume 44, Number 4, October 2007, Pages 515-539

S 0273-0979(07)01175-5

Article electronically published on June 26, 2007

\title{
EULER AND HIS WORK ON INFINITE SERIES
}

\author{
V. S. VARADARAJAN
}

For the $300^{\text {th }}$ anniversary of Leonhard Euler's birth

TABLE OF CONTENTS

1. Introduction

2. Zeta values

3. Divergent series

4. Summation formula

5. Concluding remarks

\section{INTRODUCTION}

Leonhard Euler is one of the greatest and most astounding icons in the history of science. His work, dating back to the early eighteenth century, is still with us, very much alive and generating intense interest. Like Shakespeare and Mozart, he has remained fresh and captivating because of his personality as well as his ideas and achievements in mathematics. The reasons for this phenomenon lie in his universality, his uniqueness, and the immense output he left behind in papers, correspondence, diaries, and other memorabilia. Opera Omnia [E], his collected works and correspondence, is still in the process of completion, close to eighty volumes and 31,000+ pages and counting. A volume of brief summaries of his letters runs to several hundred pages. It is hard to comprehend the prodigious energy and creativity of this man who fueled such a monumental output. Even more remarkable, and in stark contrast to men like Newton and Gauss, is the sunny and equable temperament that informed all of his work, his correspondence, and his interactions with other people, both common and scientific. It was often said of him that he did mathematics as other people breathed, effortlessly and continuously. It was also said (by Laplace) that all mathematicians were his students.

It is appropriate in this, the tercentennial year of his birth, to revisit him and survey his work, its offshoots, and the remarkable vitality of his themes which are still flourishing, and to immerse ourselves once again in the universe of ideas that he has created. This is not a task for a single individual, and appropriately enough, a number of mathematicians are attempting to do this and present a picture of his work and its modern resonances to the general mathematical community. To be honest, such a project is Himalayan in its scope, and it is impossible to do full justice to it. In the following pages I shall try to make a very small contribution to this project, discussing in a sketchy manner Euler's work on infinite series and its modern outgrowths. My aim is to acquaint the generic mathematician with

Received by the editors April 20, 2007 and, in revised form, April 23, 2007.

2000 Mathematics Subject Classification. Primary 01A50, 40G10, 11M99.

(C)2007 American Mathematical Society 
some Eulerian themes and point out that some of them are still awaiting complete understanding. Above all, it is the freedom and imagination with which Euler operates that are most compelling, and I would hope that the remarks below have captured at least some of it. For a tribute to this facet of Euler's work, see [C].

The literature on Euler, both personal and mathematical, is huge. The references given at the end are just a fraction of what is relevant and are in no way intended to be complete. However, many of the points examined in this article are treated at much greater length in my book $[\mathrm{V}]$, which contains more detailed references. After the book came out, Professor Pierre Deligne, of the Institute for Advanced Study, Princeton, wrote to me some letters in which he discussed his views on some of the themes treated in my book. I have taken the liberty of including here some of his comments that have enriched my understanding of Euler's work, especially on infinite series. I wish to thank Professor Deligne for his generosity in sharing his ideas with me and for giving me permission to discuss them here. I also wish to thank Professor Trond Digernes of the University of Trondheim, Norway, for helping me with electronic computations concerning some continued fractions that come up in Euler's work on summing the factorial-like series.

\section{Zeta VALUES}

Euler must be regarded as the first master of the theory of infinite series. He created it and was by far its greatest master. Perhaps only Jacobi and Ramanujan may be regarded as being even close. Before Euler entered the mathematical scene, infinite series had been considered by many mathematicians, going back to very early times. However there was no systematic theory; people had only very informal ideas about convergence and divergence. Also most of the series considered had only positive terms. Archimedes used the geometric series

$$
\frac{4}{3}=1+\frac{1}{4}+\frac{1}{4^{2}}+\frac{1}{4^{3}}+\ldots
$$

in computing, by what he called the method of exhaustion, the area cut off by a secant from a parabola. Leibniz, Gregory, and Newton had also considered various special series, among which the Leibniz evaluation,

$$
\frac{\pi}{4}=1-\frac{1}{3}+\frac{1}{5}-\frac{1}{7}+\ldots,
$$

was a most striking one. In the fourteenth century people discussed the harmonic series

$$
1+\frac{1}{2}+\frac{1}{3}+\ldots
$$

and Pietro Mengoli (1625-1686) seems to have posed the problem of finding the sum of the series

$$
1+\frac{1}{2^{2}}+\frac{1}{3^{2}}+\ldots
$$

This problem generated intense interest, and the Bernoulli brothers, Johann and Jakob, especially the former, appear to have made efforts to find the sum. It came to be known as the Basel problem. But all efforts to solve it had proven useless, and even an accurate numerical evaluation was extremely difficult because of the slow decay of the terms. Indeed, since

$$
\frac{1}{n}-\frac{1}{n+1}=\frac{1}{n(n+1)}<\frac{1}{n^{2}}<\frac{1}{n(n-1)}=\frac{1}{n-1}-\frac{1}{n}
$$


we have

$$
\frac{1}{N+1}<\sum_{n=N+1}^{\infty} \frac{1}{n^{2}}<\frac{1}{N}
$$

so that to compute directly the sum with an accuracy of six decimal places would require taking into account at least a million terms.

Euler's first attack on the Basel problem already revealed how far ahead of everyone else he was. Since the terms of the series decreased very slowly, Euler realized that he had to transform the series into a rapidly convergent one to facilitate easy numerical computation. He did exactly that. To describe his result, let me use modern notation (for brevity) and write

$$
\zeta(2)=1+\frac{1}{2^{2}}+\frac{1}{3^{2}}+\ldots
$$

Then Euler's remarkable formula is

$$
\zeta(2)=(\log 2)^{2}+2 \sum_{n=1}^{\infty} \frac{1}{n^{2} \cdot 2^{n}}
$$

with

$$
\log 2=\frac{1}{2}+\frac{1}{8}+\frac{1}{24}+\cdots=\sum_{n=1}^{\infty} \frac{1}{n \cdot 2^{n}} .
$$

The terms in the series are geometric, and the one for $\log 2$ is obtained by taking the value $x=\frac{1}{2}$ in the power series for $-\log (1-x)$. However formula (1) lies deeper. Using this he calculated $\zeta(2)$ accurately to six places and obtained the value

$$
\zeta(2)=1.644944 \ldots
$$

To derive (1) Euler introduced the power series

$$
x+\frac{x^{2}}{2^{2}}+\frac{x^{3}}{3^{2}}+\cdots=\sum_{n=1}^{\infty} \frac{x^{n}}{n^{2}},
$$

which is the generating function of the sequence $\left(1 / n^{2}\right)$. This is an idea of great significance for him because, throughout his life, especially when he was attempting to build a theory of divergent series, he regarded infinite series as arising out of generating functions by evaluation at special values. In this case the function in question has an integral representation: namely

$$
\sum_{n=1}^{\infty} \frac{x^{n}}{n^{2}}=\operatorname{Li}_{2}(x)
$$

where

$$
\operatorname{Li}_{2}(x):=\int_{0}^{x} \frac{-\log (1-t)}{t} d t=\iint_{0<t_{2}<t_{1}<x} \frac{d t_{1} d t_{2}}{t_{1}\left(1-t_{2}\right)} .
$$

It is the first appearance of the dilogarithm, a special case of the polylogarithms which have been studied recently in connection with multizeta values (more about these later). Clearly

$$
\zeta(2)=\mathrm{Li}_{2}(1) \text {. }
$$

The integral representation allowed Euler to transform the series as we shall see now. He obtained the functional equation

$$
\mathrm{Li}_{2}(x)+\mathrm{Li}_{2}(1-x)=-\log x \log (1-x)+\mathrm{Li}_{2}(1),
$$


which leads, on taking $x=\frac{1}{2}$, to

$$
\zeta(2)=(\log 2)^{2}+2 \sum_{n=1}^{\infty} \frac{1}{n^{2} \cdot 2^{n}} .
$$

The formula (3) is easy to prove. We write

$$
\zeta(2)=\int_{0}^{u} \frac{-\log (1-x)}{x} d x+\int_{u}^{1} \frac{-\log (1-x)}{x} d x .
$$

We then change $x$ to $1-x$ in the second integral and integrate it by parts to get (1). More than the specific result, the significance of Euler's result lies in the fact that it lifted the entire theory of infinite series to a new level and brought new ideas and themes.

Still Euler was not satisfied, since he was far from an exact evaluation. Then suddenly, he had an idea which led him to the goal. In his paper that gave this new method for the solution of the explicit evaluation he writes excitedly at the beginning: So much work has been done on the series $\zeta(n)$ that it seems hardly likely that anything new about them may still turn up.... I, too, in spite of repeated efforts, could achieve nothing more than approximate values for their sums... Now, however, quite unexpectedly, I have found an elegant formula for $\zeta(2)$, depending on the quadrature of a circle [i.e., upon $\pi$ ] (from Andre Weil's translation).

Euler's idea was based on an audacious generalization of Newton's formula for the sums of powers of the roots of a polynomial to the case when the polynomial was replaced by a power series. Writing a polynomial in the form

$$
1-\alpha s+\beta s^{2}-\cdots \pm \rho s^{k}=\left(1-\frac{s}{a}\right)\left(1-\frac{s}{b}\right) \cdots\left(1-\frac{s}{r}\right)
$$

we have

$$
\alpha=\frac{1}{a}+\frac{1}{b}+\cdots+\frac{1}{r}, \quad \beta=\frac{1}{a b}+\frac{1}{a c}+\ldots
$$

and so on. In particular

$$
\frac{1}{a^{2}}+\frac{1}{b^{2}}+\cdots+\frac{1}{r^{2}}=\alpha^{2}-2 \beta
$$

and more generally

$$
S_{3}=\alpha^{3}-3 \alpha \beta+3 \gamma, S_{4}=\alpha^{4}-4 \alpha^{2} \beta+4 \alpha \gamma+2 \beta^{2}-4 \delta
$$

and so on, where

$$
S_{k}=\frac{1}{a^{k}}+\frac{1}{b^{k}}+\cdots+\frac{1}{r^{k}} .
$$

Euler's idea was to apply these relations wholesale to the case when the polynomial is replaced by a power series

$$
1-\alpha s+\beta s^{2}-\ldots
$$

indeed, to the power series

$$
1-\sin s=1-s+\frac{s^{3}}{6}-\ldots
$$

The function $1-\sin s$ has the roots (all roots are double)

$$
\frac{\pi}{2}, \frac{\pi}{2},-\frac{3 \pi}{2},-\frac{3 \pi}{2}, \frac{5 \pi}{2}, \frac{5 \pi}{2}, \ldots,
$$


and so the above formulas give the following. First,

$$
\frac{4}{\pi}\left(1-\frac{1}{3}+\frac{1}{5}-\ldots\right)=1
$$

which is Leibniz's result. But now one can keep going and get

$$
\frac{8}{\pi^{2}}\left(1+\frac{1}{3^{2}}+\frac{1}{5^{2}}+\ldots\right)=1
$$

which leads at once to

$$
\zeta(2)=\frac{\pi^{2}}{6}
$$

One can go on and on, which is what Euler did, calculating $\zeta(2 k)$ up to $2 k=12$. In particular

$$
\zeta(4)=1+\frac{1}{2^{4}}+\frac{1}{3^{4}}+\cdots=\frac{\pi^{4}}{90} .
$$

The same method can be applied to $\sin s$ and leads to the same results.

Euler communicated these (and other) results to his friends (the Bernoullis in particular), and very soon everyone that mattered knew of Euler's sensational discoveries. He knew that his derivations were open to serious objections, many of which he himself was aware of. The most important of the objections were the following: (1) How can one be sure that $1-\sin s$ does not have other roots besides the ones written? (2) If $f(s)$ is any function to which this method is applied, $f(s)$ and $e^{s} f(s)$ both have the same roots and yet they should lead to different formulae. Nevertheless the numerical evaluations bolstered Euler's confidence, and he kept working to achieve a demonstration that would satisfy his critics. It took him about ten years, but he finally succeeded in obtaining the famous product formula for $\sin s$ :

$$
\frac{\sin x}{x}=\prod_{n=1}^{\infty}\left(1-\frac{x^{2}}{n^{2} \pi^{2}}\right) .
$$

Once this formula is established, all the objections disappear, as he himself remarked.

The proof of (4) by Euler was beautiful and direct. He wrote

$$
\frac{\sin x}{x}=\lim _{n \rightarrow \infty} \frac{\left(1+\frac{i x}{n}\right)^{n}-\left(1-\frac{i x}{n}\right)^{n}}{2 i x}
$$

and factorized explicitly the polynomials

$$
q_{n}(x):=\frac{\left(1+\frac{i x}{n}\right)^{n}-\left(1-\frac{i x}{n}\right)^{n}}{2 i x}
$$

to get

$$
q_{n}(x)=\prod_{k=1}^{p}\left(1-\frac{x^{2}}{n^{2}} \frac{1+\cos \frac{2 k \pi}{n}}{1-\cos \frac{2 k \pi}{n}}\right) \quad(n=2 p+1) .
$$

The formula (4) is obtained by letting $n$ go to $\infty$ term by term in the product. As would be natural to expect, Euler does not comment on this passage to the limit; a modern rigorous argument would add just the observation that the passage to the 
limit termwise can be justified by uniform convergence, as can be seen from the easily established estimate

$$
\left|\frac{1+\cos \frac{2 k \pi}{n}}{1-\cos \frac{2 k \pi}{n}}\right| \leq C \frac{x^{2}}{k^{2} \pi^{2}}
$$

where $C$ is an absolute constant. The method is applicable to a whole slew of trigonometric as well as hyperbolic functions and allowed Euler to reach all the formulae obtained earlier by his questionable use of Newton's theorem. Among these are

$$
1-\frac{\sin s}{\sin \sigma}=\prod_{n=-\infty}^{\infty}\left(1-\frac{s}{2 n \pi+\sigma}\right)\left(1-\frac{s}{2 n \pi+\pi-\sigma}\right) .
$$

For convergence purposes this should be rewritten as

$$
\begin{aligned}
1-\frac{\sin s}{\sin \sigma} & =\left(1-\frac{s}{\sigma}\right) \prod_{n=1}^{\infty}\left(1-\frac{s}{2 n \pi+\sigma}\right)\left(1+\frac{s}{2 n \pi-\sigma}\right) \\
& \times \quad \prod_{n=1}^{\infty}\left(1-\frac{s}{(2 n-1) \pi-\sigma}\right)\left(1+\frac{s}{(2 n-1) \pi+\sigma}\right) .
\end{aligned}
$$

From the product formula (4) one can calculate by Newton's method the values of $\zeta(2 k)$ explicitly; there are no problems (as everything in sight is absolutely convergent), and Euler did this. These evaluations, especially the value

$$
\zeta(12)=\frac{691}{6825 \times 93555} \pi^{12}
$$

must have suggested to him that the Bernoulli numbers were lurking around the corner here, since

$$
B_{12}=-\frac{691}{2730} \text {. }
$$

Euler then succeeded in getting a closed formula for all the $\zeta(2 k)$.

The main idea is to logarithmically differentiate (4) (as was also observed immediately by Nicholas Bernoulli) to get (replacing $x$ by $s \pi$ )

$$
\pi \cot s \pi=\frac{1}{s}+\sum_{n=1}^{\infty}\left(\frac{1}{n+s}-\frac{1}{n-s}\right) \quad(0<s<1) .
$$

The formula is written in such a way that absolute convergence is manifest; Euler did not bother with such niceties and wrote it as

$$
\pi \cot s \pi=\sum_{-\infty}^{\infty} \frac{1}{s+n} .
$$

It is definitely more convenient to do this, interpreting the sum as a principal value. We shall do so from now on, omitting the reference to principal values for brevity. Expressing the cotangent in terms of exponentials leads one to the function

$$
B(s)=B(-s):=\frac{s}{e^{s}-1}-1+\frac{1}{2} s=\sum_{k=1}^{\infty} \frac{B_{2 k}}{(2 k) !} s^{2 k} .
$$


The $B_{2 k}$ are the Bernoulli numbers, introduced by Jakob Bernoulli many years before Euler; Euler suggested they be called Bernoulli numbers. For the first few we have

$$
B_{2}=\frac{1}{6}, B_{4}=-\frac{1}{30}, B_{6}=\frac{1}{42}, B_{8}=-\frac{1}{30}, B_{10}=\frac{5}{66}, B_{12}=-\frac{691}{2730} .
$$

Then

$$
\pi \cot s \pi-\frac{1}{s}=\frac{2 \pi i}{2 \pi i s} B(2 \pi i s)=2 \pi i \sum_{k=1}^{\infty} B_{2 k} \frac{(2 \pi i s)^{2 k-1}}{(2 k) !} .
$$

Calculating derivatives at $s=0$ we get Euler's surpassingly beautiful formula

$$
\zeta(2 k)=\frac{(-1)^{k-1} 2^{2 k-1} B_{2 k}}{(2 k) !} \pi^{2 k} .
$$

Nowadays it is customary to treat $s$ as a complex variable and establish (5) or (6) by complex methods, using periodicity and Liouville's theorem. I think however that Euler's method is unrivaled in its originality and directness. For a treatment of these formulae that is very close to Euler's and even more elementary in the sense that one works entirely over the real field, see Omar Hijab's very nice book Hi]. One should also note that the results of Euler may be viewed as the forerunners of the work of Weierstrass and Jacobi, of infinite products with specified zeros and poles, with sums over lattices in the complex plane replacing sums over integers $(\wp$ and $\vartheta$-functions).

In addition to the zeta values Euler also determined the values

$$
L(2 k+1)=1-\frac{1}{3^{2 k+1}}+\frac{1}{5^{2 k+1}}-\ldots
$$

These are the very first examples of twisting, namely replacing a series by one where the coefficients are multiplied by a character $\bmod N$ :

$$
\sum_{n \geq 1} \frac{a_{n}}{n^{s}} \longmapsto \sum_{n \geq 1} \frac{a_{n} \chi(n)}{n^{s}}
$$

where $\chi$ is a character $\bmod N$, more generally a function of period $N$. The transition from $\zeta$ to $L$ corresponds to a character $\bmod 4$ :

$$
\chi(n)= \begin{cases}(-1)^{(n-1) / 2} & \text { if } n \text { is odd } \\ 0 & \text { if } n \text { is even. }\end{cases}
$$

I shall talk more about these when I discuss Euler products. The method for the sums $L(2 k+1)$ is the same as for the zeta values and starts with the partial fraction

$$
\frac{\pi}{\sin s \pi}=\sum_{-\infty}^{\infty}(-1)^{n} \frac{1}{s+n}
$$

obtained by logarithmically differentiating the infinite product

$$
1-\frac{\sin x}{\sin s}=\prod_{n=-\infty}^{\infty}\left(1-\frac{x}{2 n \pi+s}\right)\left(1-\frac{x}{2 n \pi+\pi-s}\right)
$$

at $x=0$ and then changing $s$ to $s \pi$.

It was natural for Euler to explore if the partial fraction expansions

$$
\frac{\pi}{\sin s \pi}=\sum_{-\infty}^{\infty}(-1)^{n} \frac{1}{s+n}, \quad \pi \cot s \pi=\sum_{-\infty}^{\infty} \frac{1}{s+n}
$$


could be established by other methods. This he did in several beautiful papers, and his derivations take us through a whole collection of beautiful formulae in integral calculus, including the entire basic theory of what Legendre would later call the Eulerian integrals of the first and second kind, namely, the theory of the beta and gamma functions.

The starting point of the new derivation is the pair of formulae

$$
\begin{aligned}
& \int_{0}^{1} \frac{x^{p-1}+x^{q-p-1}}{1+x^{q}} d x=\sum_{-\infty}^{\infty}(-1)^{n} \frac{1}{p+n q} \quad(q>p>0) \\
& \int_{0}^{1} \frac{x^{p-1}-x^{q-p-1}}{1-x^{q}} d x=\sum_{-\infty}^{\infty} \frac{1}{p+n q} \quad(q>p>0) .
\end{aligned}
$$

These are derived by expanding

$$
\frac{1}{1 \pm x^{q}}
$$

as power series and integrating term by term. One has to be a bit careful in the second of these formulae since the integrals do not converge separately. It is then a question of evaluating the integrals directly to obtain the formulae

$$
\begin{aligned}
& \int_{0}^{1} \frac{x^{p-1}+x^{q-p-1}}{1+x^{q}} d x=\frac{\pi}{q \sin (p / q) \pi} \quad(q>p>0) \\
& \int_{0}^{1} \frac{x^{p-1}-x^{q-p-1}}{1-x^{q}} d x=\frac{\pi \cot (p / q) \pi}{q} \quad(q>p>0) .
\end{aligned}
$$

We then obtain (7) with $s=p / q$. For Euler this was sufficient; we would add to his derivation a remark about justifying the continuity of both sides of the formulae in $s$.

For proving (10) Euler developed a method based on a beautiful generalization of the familiar formula (indefinite integration)

$$
\int \frac{d x}{1+x^{2}}=\arctan x
$$

Euler obtains for

$$
\int_{0}^{x} \frac{x^{m-1}}{1+x^{2 n}} d x \quad(2 m>n>0 . m, n \text { integers })
$$

the formula

$$
\begin{aligned}
& \frac{(-1)^{m-1}}{2 n} \sum_{k=1}^{n} \cos (2 k-1) m \frac{\pi}{2 n} \log \left(1+2 x \cos (2 k-1) \frac{\pi}{2 n}+x^{2}\right) \\
& \quad+\frac{(-1)^{m-1}}{n} \sum_{k=1}^{n} \sin (2 k-1) m \frac{\pi}{2 n} \arctan \frac{x \sin (2 k-1) \frac{\pi}{2 n}}{1+x \cos (2 k-1) \frac{\pi}{2 n}} .
\end{aligned}
$$

The formula is obtained using partial fractions and the factorization of $\left(1+x^{2 n}\right)$. We now let $x \rightarrow \infty$ in this formula. Using the identities (which Euler derived as 
special cases of a whole class of trigonometric identities)

$$
\begin{gathered}
\sum_{k=1}^{n} \cos \frac{(2 k-1) m \pi}{2 n}=0 \\
\sum_{k=1}^{n}(2 k-1) \sin \frac{(2 k-1) m \pi}{2 n}=\frac{(-1)^{m-1} n}{\sin \frac{m \pi}{2 n}},
\end{gathered}
$$

we get, with Euler,

$$
\int_{0}^{\infty} \frac{x^{m-1}}{1+x^{2 n}} d x=\frac{\pi}{2 n \sin \frac{m \pi}{2 n}} .
$$

We put $p=m, q=2 n$ and rewrite this as

$$
\int_{0}^{\infty} \frac{x^{p-1}}{1+x^{q}} d x=\frac{\pi}{q \sin \frac{p \pi}{q}} \quad(q>p>0) .
$$

Here $q$ is even; but if $q$ is odd, the substitution $x=y^{2}$ changes the integral to one with the even integer $2 q$, and we obtain the above formula for odd $q$ also. Euler does not stop with this of course; he goes on to evaluate all the integrals of the form

$$
\int_{0}^{\infty} \frac{x^{p-1}}{\left(1+x^{q}\right)^{k}} d x
$$

In particular he finds

$$
\int_{0}^{\infty} \frac{x^{m-1}}{1-2 x^{n} \cos \omega+x^{2 n}} d x=\frac{\pi \sin \frac{n-m}{n}(\pi-\omega)}{n \sin \omega \sin \frac{(n-m) \pi}{n}} .
$$

For $\omega=\frac{\pi}{2}$ this reduces to the previous formula.

The derivation of the second integral in (10) is similar but more complicated since we have to take into account the fact that the integrals do not converge separately. It is based on getting a formula for

$$
\int_{0}^{x} \frac{x^{m-1}}{1-x^{2 n}} d x
$$

and we omit the details. The result is

$$
\int_{0}^{1} \frac{x^{m-1}-x^{2 n-m-1}}{1-x^{2 n}} d x=\frac{\pi}{2 n} \cot \frac{m \pi}{2 n},
$$

which leads as before to the second formula in (10). It is to be noted that in this method also the factorization of $\left(1 \pm x^{2 n}\right)$ enters decisively, exactly as in his original proof of the infinite product for $\sin x$.

Finally one could also obtain (10) as a consequence of the theory of the gamma function, using only formulae that were known to Euler. We are used to writing

$$
\Gamma(s+1)=\int_{0}^{\infty} e^{-x} x^{s} d x
$$

but Euler always preferred to write it as

$$
[s]=s !=\int_{0}^{1}(-\log x)^{s} d x
$$

and think of it as an interpolation for $n$ ! He knew the functional equation

$$
[s]=(s+1)[s-1]
$$


as well as the formula

$$
\Gamma(s) \Gamma(1-s)=\frac{\pi}{\sin s \pi},
$$

(the corollary)

$$
\Gamma\left(\frac{1}{2}\right)=\sqrt{\pi}
$$

and the limit formula

$$
\Gamma(1+m)=\lim _{m \rightarrow \infty} \frac{1.2 \ldots n}{(m+1)(m+2) \ldots(m+n)}(n+1)^{m},
$$

which he would write as

$$
[m]=\lim _{m \rightarrow \infty} \frac{1.2^{m}}{m+1} \frac{2^{1-m} \cdot 3^{m}}{m+2} \ldots \frac{n^{1-m}(n+1)^{m}}{m+n} .
$$

In fact it is in this form he introduces the Gamma function in one of his early letters to Goldbach. The derivation of (10) is now a straightforward consequence of the theory of these integrals. One gets

$$
\int_{0}^{1} \frac{x^{q-p-1}}{1+x^{q}} d x=\int_{1}^{\infty} \frac{x^{p-1}}{1+x^{q}} d x
$$

so that

$$
\int_{0}^{1} \frac{x^{p-1}+x^{q-p-1}}{1+x^{q}} d x=\int_{0}^{\infty} \frac{x^{p-1}}{1+x^{q}} d x=\frac{1}{q} B\left(\frac{p}{q}, 1-\frac{p}{q}\right)=\frac{\pi}{q \sin (p / q) \pi} .
$$

Once again the treatment of the second integral in (10) is more delicate.

The partial fractions (9) can be differentiated and specialized to yield explicit values for many infinite series. Euler worked out a whole host of these, with or without the twisting mentioned earlier. The sums he treated are of the form

$$
\sum_{n \in \mathbf{Z}} \frac{h(n)}{(n q+p)^{r}}
$$

where $h$ is a periodic function, and their values are of the form

$$
g \pi^{r}
$$

where $g$ is a cyclotomic number. The series he obtains are actually Dirichlet series corresponding to various characters $\bmod q$ and their variants. Thus, with $\chi$ as the non-trivial character mod 3 , extended to $\mathbf{Z}$ by 0 ,

$$
\frac{2 \pi}{3 \sqrt{3}}=\sum_{n=1}^{\infty} \frac{(-1)^{n-1} \chi(n)}{n}, \quad \frac{\pi}{3 \sqrt{3}}=\sum_{n=1}^{\infty} \frac{\chi(n)}{n},
$$

which he would write as

$$
\begin{aligned}
& \frac{2 \pi}{3 \sqrt{3}}=1+\frac{1}{2}-\frac{1}{4}-\frac{1}{5}+\frac{1}{7}+\frac{1}{8}-\frac{1}{10}-\frac{1}{11}+\ldots \\
& \frac{\pi}{3 \sqrt{3}}=1-\frac{1}{2}+\frac{1}{4}-\frac{1}{5}+\frac{1}{7}-\frac{1}{8}+\ldots
\end{aligned}
$$

Also

$$
\frac{\pi^{2}}{8 \sqrt{2}}=\sum_{n=1}^{\infty} \frac{\chi_{8}(n)}{n^{2}} \quad \frac{\pi^{2}}{6 \sqrt{3}}=\sum_{n=1}^{\infty} \frac{\chi_{12}(n)}{n^{2}}
$$


where

$$
\chi_{8}(n)=\left\{\begin{array}{ll}
+1 & \text { if } n \equiv \pm 1 \bmod 8 \\
-1 & \text { if } n \equiv \pm 3 \bmod 8 \\
0 & \text { if otherwise }
\end{array} \quad \chi_{12}(n)= \begin{cases}+1 & \text { if } n \equiv \pm 1 \bmod 12 \\
-1 & \text { if } n \equiv \pm 5 \bmod 12 \\
0 & \text { if otherwise, }\end{cases}\right.
$$

which he would write as

$$
\begin{aligned}
& \frac{\pi^{2}}{8 \sqrt{2}}=1-\frac{1}{3^{2}}-\frac{1}{5^{2}}+\frac{1}{7^{2}}+\ldots \\
& \frac{\pi^{2}}{6 \sqrt{3}}=1-\frac{1}{5^{2}}-\frac{1}{7^{2}}+\frac{1}{11^{2}}+\ldots
\end{aligned}
$$

and so on.

Multizeta values. Throughout his life Euler tried to determine the zeta values at odd integers, $\zeta(3), \zeta(5), \ldots$ but was unsuccessful. He obtained many formulae linking them but was unable to get a breakthrough. Late in his life, almost thirty years after his discoveries, he wrote a beautiful paper where he introduced what are now called multizeta values. The double zeta values are nowadays defined as

$$
\zeta(a, b)=\sum_{m>n>0} \frac{1}{m^{a} n^{b}} \quad(a, b \in \mathbf{Z}, a \geq 2, b \geq 1) .
$$

This is a slight variant of Euler's definition which we write as $\zeta_{E}(a, b)$, in which he would sum for $m \geq n$ and write the sum as

$$
1+\frac{1}{2^{a}}\left(1+\frac{1}{2^{b}}\right)+\frac{1}{3^{a}}\left(1+\frac{1}{2^{b}}+\frac{1}{3^{b}}\right)+\ldots
$$

so that

$$
\zeta_{E}(a, b)=\zeta(a, b)+\zeta(a+b) .
$$

He proved the beautiful relation

$$
\zeta(2,1)=\zeta(3)
$$

as well as the more general

$$
\zeta(p, 1)+\zeta(p-1,2)+\cdots+\zeta(2, p-1)=\zeta(p+1)
$$

from which he derived the relations

$$
2 \zeta(p-1,1)=(p-1) \zeta(p)-\sum_{2 \leq q \leq p-2} \zeta(q) \zeta(p-q) .
$$

In recent years people have defined the multizeta values by

$$
\zeta\left(s_{1}, s_{2}, \ldots, s_{r}\right)=\sum_{n_{1}>n_{2}>\cdots>n_{r}>0} \frac{1}{n_{1}^{s_{1}} n_{2}^{s_{2}} \ldots n_{r}^{s_{r}}} \quad\left(s_{i} \in \mathbf{Z}, s_{1} \geq 2, s_{i} \geq 1\right) .
$$

The Euler identities have been generalized, new identities have been discovered by Ecalle and others, and considerable progress has been made about the nature of these numbers, including the odd zeta values. I mention the results that $\zeta(3)$ is irrational [A], that an infinity of the odd zeta values are irrational $[\overrightarrow{B R}$, and that at least one of $\zeta(5), \zeta(7), \ldots, \zeta(21)$ is irrational $[\mathrm{R}]$. The multizeta values have been interpreted as period integrals, and this interpretation may possibly lead to a better understanding of them [KZ], [D1]. For more details and references see [V]. 


\section{Divergent SERIES}

It was in the systematic theory of infinite series (beyond explicit evaluations) that Euler made one of his greatest contributions, namely his creation of a theory of divergent series, or at least the first steps towards such a theory. He knew that by attempting to associate numbers as values of diverging series he was going beyond the confines of what people were used to thinking about, and yet he insisted that one has to build a theory of divergent series in order to free analytical methods from artificial limitations. There is no better place to hear his viewpoint than from his great 1760 paper (communicated in 1755) De seriebus divergentibus [E1]:

Notable enough, however, are the controversies over the series $1-1+1-1+1-1+\ldots$ whose sum was given by Leibniz as $1 / 2$, although others disagree. No one has yet assigned another value to that sum, and so the controversy turns on the question whether the series of this type have a certain sum. Understanding of this question is to be sought in the word "sum"; this idea, if thus conceived-namely the sum of a series is said to be that quantity to which it is brought closer as more terms of the series are taken-has relevance only for convergent series, and we should in general give up this idea of sum for divergent series. Wherefore, those who thus define a sum cannot be blamed if they claim they are unable to assign a sum to a series. On the other hand, as series in analysis arise from the expansion of fractions or irrational quantities or even transcendentals, it will in turn be permissible in calculation to substitute in place of such a series that quantity out of whose development it is produced. For this reason, if we employ this definition of sum, that is to say, the sum of a series is that quantity which generates the series, all doubts with respect to divergent series vanish and no further controversy remains on this score, in as much as this definition is applicable equally to convergent or divergent series. Accordingly, Leibniz, without any hesitation, accepted for the series $1-1+1-1+1-1+\ldots$, the sum $1 / 2$, which arises out of the expansion of the fraction $1 / 1+1$, and for the series $1-2+3-4+5-6+\ldots$, the sum $1 / 4$, which arises out of the expansion of the formula $1 /(1+1)^{2}$. In a similar way a decision for all divergent series will be reached, where always a closed formula from whose expansion the series arises should be investigated. However,

it can happen very often that this formula itself is difficult to find, as here where the author treats an exceptional example, that divergent series par excellence $1-1+2-6+24-120+720-5040+\ldots$, which is Wallis' hypergeometric series, set out with alternating signs; this series, in whatever formula it finds its origin and however much this formula is valid, is seen to be determinable by only the deepest study of higher Analysis. Finally, after various attempts, the author by a wholly singular method using continued fractions found that the sum of this series is about 0.596347362123 , and in this decimal fraction the error does not affect even the last digit. Then he proceeds to other similar series of wider application and he explains how to assign them a sum in the same way, where the word "sum" 
has that meaning which he has here established and by which all controversies are cut off. (From the translation of E. J. Barbeau and P. J. Leah $[\mathrm{BL}]$

The paper shows clearly the fact that he understood what was involved, that the number we associate to an infinite series is a matter of convention, and that a systematic treatment of this question would be very beneficial to the development of Analysis.

This paper is only a culmination of his ideas on the subject which had been in gestation for many years prior to its communication. In 1745 he had discussed these matters in letters to Goldbach and Nicholas Bernoulli, especially the summing of the factorial series

$$
\sum_{n \geq 0}(-1)^{n} n !
$$

which he called the divergent series par excellence. In it Euler has this to say (free translation from German):

... I believe that every series should be assigned a certain value. However, to account for all the difficulties that have been pointed out in this connection, this value should not be denoted by the name sum, because usually this word is connected with the notion that a sum has been obtained by a real summation: this idea however is not applicable to "seriebus divergentibus"...

In the nineteenth century, with Abel, Cauchy, Dedekind, and Weierstrass, rigorous foundations were laid for Analysis, and divergent series were banned as "the work of the devil". With the rise of these formal principles Euler's reputation also suffered, and people began to misunderstand what he was trying to do and started thinking of him as a loose mathematician. It should have been clear that no one who calculated the values of many convergent series to tremendous accuracy would have loose ideas about when series diverge and what their values are. His true greatness in these matters was not appreciated till a century afterwards when a genuine theory of divergent series was created and it became clear how far he was ahead of his time $\mathrm{Ha}$, C].

It is true that Euler had some misconceptions regarding summation of divergent series. He appeared to believe that all series could be summed by some procedure or other and also that in general all summation procedures would lead to the same value. The actual theory of divergent series shows that the situation is much more subtle. However in my opinion these are differences in detail that do not alter the fact that he took the first steps in creating a true theory of divergent series.

Euler had several different methods of summing divergent series, but most of all he worked with what we now call Abel summation. If $\sum_{n>0} a_{n}$ is a series such that $\sum_{n \geq 0} a_{n} z^{n}$ converges inside the unit disk, we shall say that $\sum_{n \geq 0} a_{n}$ is Abel summable to the value $s$ if

$$
\lim _{0<x<1, x \rightarrow 1-} \sum_{n \geq 0} a_{n} x^{n}=s .
$$

In particular, if the sum $f(z)$ of the power series extends analytically to a domain containing $z=1$, we can take $f(1)$ as the value of the sum. This is what Euler did. He often referred to it as the generating function method. 
For a large class of divergent series which arise naturally in Analysis this method turns out to be adequate. Indeed, by using it Euler was able to discover the functional equation of the Riemann zeta function one hundred years before Riemann did. However for the factorial series this method would fail, as the corresponding power series has zero radius of convergence. That is why Euler called it the divergent series par excellence. His method of summing it is truly fascinating and would eventually incarnate into what we now call Borel summation, discovered by Emile Borel [Bo]. Borel summation and its generalizations have proved surprisingly powerful in many applications, such as quantum field theory and dynamical systems.

The functional equation of the zeta. The functional equation of the zeta, established by Riemann, who was the first to treat the zeta as a function of the complex variable $s$, is given by

$$
\zeta(1-s)=2(2 \pi)^{-s} \cos \frac{s \pi}{2} \Gamma(s) \zeta(s) .
$$

However Euler was led to this equation one hundred years before Riemann. Of course Euler worked with real $s$ (this was the case even for Dirichlet). Euler verified this equation exactly for all integer values of $s$ and numerically to great accuracy for many fractional values as well. For $s$ a positive integer $\geq 2,1-s$ is a negative integer and so the series for the corresponding zeta value diverges, showing that its value has to be interpreted by a summation procedure; naturally Euler used the generating function method (Abel summation). Of course we now know that with appropriate growth conditions the verification at the integers establishes the functional equation. As usual Euler worked with

$$
\eta(s)=1-2^{-s}+3^{-s}=-\cdots=\left(1-2^{1-s}\right) \zeta(s)
$$

rather than $\zeta(s)$ for better convergence and more accurate numerical evaluation. For $\eta$ the functional equation is

$$
\frac{\eta(1-s)}{\eta(s)}=-\frac{2^{s}-1}{2^{s-1}-1} \frac{\cos \frac{s \pi}{2}}{\pi^{s}} \Gamma(s) .
$$

For $s=2 k$ an even positive integer

$$
\eta(1-2 k)=1^{2 k-1}-2^{2 k-1}+3^{2 k-1}-\ldots
$$

with generating function

$$
1^{2 k-1} x-2^{2 k-1} x^{2}+3^{2 k-1} x^{3}-\ldots
$$

We now write $x=e^{-y}$ so that $x \rightarrow 1-$ corresponds to $y \rightarrow 0+$. From

$$
e^{-y}-e^{-2 y}+e^{-3 y}-\cdots=\frac{1}{e^{y}+1}
$$

we get, on differentiating repeatedly,

$$
1^{m} e^{-y}-2^{m} e^{-2 y}+3^{m} e^{-3 y}-\cdots=(-1)^{m} \frac{d^{m}}{d y^{m}}\left(\frac{1}{e^{y}+1}\right) .
$$

Since

$$
\frac{1}{e^{y}+1}=\frac{1}{2}+\sum_{k=1}^{\infty} \frac{\left(1-2^{2 k}\right) B_{2 k}}{(2 k) !} y^{2 k-1}
$$


we have

$$
\left.(-1)^{m} \frac{d^{m}}{d y^{m}}\left(\frac{1}{e^{y}+1}\right)\right|_{y=0}= \begin{cases}\frac{1}{2} & \text { if } m=0 \\ 0 & \text { if } m=2,4,6, \ldots \\ \frac{2^{2 k}-1}{2 k} B_{2 k} & \text { if } m=2 k-1 .\end{cases}
$$

Thus Euler obtained his remarkable formulae

$$
1^{m}-2^{m}+3^{m}-\cdots= \begin{cases}\frac{1}{2} & \text { if } m=0 \\ 0 & \text { if } m=2,4,6, \ldots \\ \frac{2^{2 k}-1}{2 k} B_{2 k} & \text { if } m=2 k-1 .\end{cases}
$$

In particular

giving

$$
\eta(1-2 k)=\frac{2^{2 k}-1}{2 k} B_{2 k}
$$

which is (12) for $s=2 k$. Here we have used the even zeta values computed already by him. For $s$ odd (12), after bringing $\eta(s)$ to the right side, is trivially true since both sides are 0 . This is in fact the reason why Euler substituted $\cos k \pi$ for $(-1)^{k}$ in the previous calculation, clearly suggesting that he was thinking of the functional equation for non-integral values of $s$. Euler then proceeded to write that "... I shall hazard the conjecture that the relation (12) is true for all $s . . . "$

He then proceeded to verify this conjecture as far as he could for other values of $s$. For $s=1$

$$
\eta(1)=\log 2, \quad \eta(0)=\frac{1}{2}, \quad \eta(0)=1-1+1-1+1-1+\ldots .
$$

The Leibniz series has the sum $1 / 2$. For $s=0$ the calculations are the same since the roles of $s$ and $1-s$ are reversed. Euler notes explicitly that the transformation $s \mapsto 1-s$ leaves the functional equation invariant, a fact that is easily verified and depends on the relation

$$
\Gamma(s) \Gamma(1-s)=\frac{\pi}{\sin s \pi},
$$

which he knew. Thus the functional equation was verified whenever $s$ is any integer, positive, negative, or zero.

For fractional values of $s$ Euler resorted to numerical evaluation. For $s=1 / 2$ we have $\eta(s)=\eta(1-s)=\eta(1 / 2)$, and the verification of (10) depends on the relation

$$
\Gamma\left(\frac{1}{2}\right)=\sqrt{\pi}
$$

which Euler knew. Euler treated $s=3 / 2$, and it appears that he had verified the functional equation for $s=(2 i+1) / 2(i=1,2,3,4,5,6, \ldots)$. For $s=3 / 2$ Euler used the Euler-Maclaurin sum formula to compute the sums appearing on both sides of (11). This of course is not the same thing as being summed by the generating function method, but Euler went ahead because he firmly believed that all methods of summation lead to the same value. (The Euler-Maclaurin summation method was resurrected by Ramanujan centuries later.) Much later Landau justified Euler's method of summation for all $s$. 
The summation of the factorial series. The summation of the factorial series, the divergent series par excellence as he called it, presented serious problems for Euler and his philosophy of using the generating function for summing divergent series. The formal power series

$$
f(x)=1-1 ! x+2 ! x^{2}-\cdots=\sum_{n \geq 0}(-1)^{n} n ! x^{n}
$$

does not converge anywhere and so his generating function has only a formal existence. Euler [E1] introduced the function $g(x)=x f(x)$, and his method starts with the differential equation satisfied by $g$ :

$$
x^{2} \frac{d g}{d x}+g=x,
$$

for which one can find the complete integral

$$
g(x)=e^{1 / x} \int \frac{1}{x} e^{-1 / x} d x+c e^{1 / x},
$$

$c$ being an arbitrary constant. It is very interesting to note that the function $e^{-1 / x}$ is rapidly decreasing as $x \rightarrow 0+$ and its derivatives at 0 are all 0 ; it is in fact the classical Cauchy function, which is flat at 0 . The appearance of flat functions is characteristic of the theory that Euler discovered in the process of his summing the factorial series. It is easy to see that as $x \rightarrow 0+$,

$$
e^{1 / x} \int_{0}^{x} \frac{1}{t} e^{-1 / t} d t=O(x)
$$

and so, by choosing $c=0$, we match the asymptotics of the integral with that of the formal series to get, with Euler,

$$
f(x)=\frac{1}{x} e^{1 / x} \int_{0}^{x} \frac{1}{t} e^{-1 / t} d t
$$

so that

$$
f(1)=\int_{0}^{1} \frac{e^{1-1 / t}}{t} d t,
$$

which reduces the problem to the evaluation of the integral. Today we would not write equality in (14) and (15), but use some notation such as $\sim$ to indicate that the two sides are asymptotic. Euler first evaluates the integral numerically; writing $h$ for the integrand and using the trapezoidal rule for approximating the integral by

$$
\frac{1}{10}[(1 / 2) h(0)+h(1 / 10)+h(2 / 10)+\ldots h(9 / 10)+(1 / 2) h(1)],
$$

he gets

$$
1-1 !+2 !-3 !+\cdots=0.59637255 \ldots
$$

This is a surprisingly accurate evaluation, which may be partially explained by using the Euler-Maclaurin summation formula (see below).

He was not satisfied with this and wanted a more accurate evaluation of the integral. For this purpose he discovered a continued fraction for it,

$$
f(x)=\sum_{n \geq 0}(-1)^{n} n ! x^{n}=\frac{1}{1+} \frac{x}{1+} \frac{x}{1+} \frac{2 x}{1+} \frac{2 x}{1+} \frac{3 x}{1+} \frac{3 x}{1+} \text { etc. }
$$


Using this he finds for the integral the value

$$
I=0.596347362123 \ldots(I=0.5963473625 \text { using MAPLE }) .
$$

Euler did not stop with this single example. In his letter to Nicolaus Bernoulli that I mentioned he says that similar methods can be applied to many other series and related them to corresponding continued fractions. He treated in [E1] for instance the class of series

$$
g=g_{m p q}=x^{m}-p x^{m+q}+p(p+q) x^{m+2 q}-p(p+q)(p+2 q) x^{m+3 q}-\ldots
$$

For $m=p=q=1$ one obtains the factorial series $x f=g_{111} . g$ satisfies

$$
x^{q+1} g^{\prime}+\left[(p-m) x^{q}+1\right] g=x^{m}
$$

so that (matching asymptotics at $x=0$ as before)

$$
g \sim e^{\frac{1}{q x^{q}}} x^{m-p} \int_{0}^{x} e^{-1 / q t^{q}} t^{p-q-1} d t .
$$

Euler finds for $g$ the continued fraction

$$
g_{m n}=\frac{x^{m}}{1+} \frac{p x^{q}}{1+} \frac{q x^{q}}{1+} \frac{(p+q) x^{q}}{1+} \frac{2 q x^{q}}{1+} \frac{(p+2 q) x^{q}}{1+} \frac{3 q x^{q}}{1+} \frac{\left(p+3 q x^{q}\right)}{1+} \text { etc. }
$$

If we take $p=m=1$ and $q=2$, we get the series

$$
g=x-1 x^{3}+1.3 x^{5}-1.3 .5 x^{7}+1.3 .5 .7 x^{9}-\ldots .
$$

For $g$ the integral is given by

$$
g \sim e^{\frac{1}{2 x^{2}}} \int_{0}^{x} e^{-1 / 2 t^{2}} \frac{1}{t^{2}} d t .
$$

Thus he finds

$$
1-1+1.3-1.3 .5+1.3 .5 .7-\cdots=\int_{0}^{1} e^{\left[1-\left(1 / t^{2}\right)\right] / 2} \frac{1}{t^{2}} d t
$$

as well as the continued fraction

$$
\frac{1}{1+} \frac{1}{1+} \frac{2}{1+} \frac{3}{1+} \frac{4}{1+} \frac{5}{1+} \text { etc. }
$$

Using the same methods as before he finds for this series the value

$$
0.65568 \text { (0.6556795424 using MAPLE). }
$$

It is important to note that these continued fractions of Euler are quite different from the classical simple continued fractions which are of the form

$$
a_{0}+\frac{1}{a_{1}+} \frac{1}{a_{2}+} \ldots
$$

where the $a$ 's are positive integers. The Eulerian ones are of the form

$$
\frac{1}{1+} \frac{a_{1}}{1+} \frac{a_{2}}{1+} \ldots
$$

where the $a_{i}$ are positive integers. In his treatment Euler implicitly assumes that these behave like the simple ones. It can be shown that Euler's assumption is in fact true and that these continued fractions do converge, at least when $a_{n}=O(n)$, the partial convergents being alternately above and below the true value. The nature of the values of these continued fractions (algebraic, transcendental) is not known. Calculating with Maple, we find that the limiting value of the continued fractions of Euler is accurate to a huge number of decimal places. Restricted to 
hand computing, Euler resorted to a method of calculating which produced a result accurate to about 8 decimal places. To explain his method let us think of the finite continued fractions as the result of applying a sequence of fractional linear transformations. Thus

$$
\frac{1}{1+} \frac{a_{1}}{1+} \frac{a_{2}}{1+} \ldots \frac{a_{n-1}}{1+} \frac{a_{n}}{1+t}=F_{a_{1}} F_{a_{2}} \ldots F_{a_{n}}(t)=: H_{n}(t)
$$

where $F_{a}$ is the map $z \longmapsto a / 1+z$. Euler's idea was to evaluate, for a moderately large $N, H_{N}(t)=F_{1} F_{2} \ldots F_{N}(t)$ not at $t=0$ but at a suitable point $t=t_{N}$, a step that is justifiable in view of the following result that can be proved: when $x_{n}=O(n)$, for any arbitrary sequence $\left(t_{n}\right), t_{n} \in[0, \infty]$, the sequence $H_{n}\left(t_{n}\right)$ has a limit as $n \rightarrow \infty$, and this limit is independent of the sequence, equal for example to the limit when we choose $t_{n}=0$ for all $n$. The idea behind evaluating $H_{N}(t)$ at $t=t_{N}$ instead of $t=0$ is to improve the rapidity of convergence to the limit. Euler starts with the choice of $t_{N}$ as the fixed point of $F_{N+1}$. For $N=20$ this gave for Euler a value accurate to about 4 decimals, but then, remarkably, he makes a further variation in the choice of $t_{N}$ that gets him to about 8 decimal place accuracy (see $[\mathrm{BL}]$ ). One can explain his method and even improve on it, as was shown to me by Deligne [D], but it would take me too far afield to do it here. I hope however to discuss these matters in more detail on a later occasion DV].

In the theory of summability of divergent series that grew in the nineteenth century, it is the theory of Borel summation that allows us to put Euler's ideas on the summation of factorial-like series into proper perspective. The Borel transform $f^{\sim}$ of the formal power series $f=\sum_{n \geq 0} a_{n} z^{n}$ is the formal power series

$$
f^{\sim}(z)=\sum_{n \geq 0} a_{n} \frac{z^{n}}{n !} .
$$

At a completely formal level we can then recover the original series by the formula

$$
\sum_{n \geq 0} a_{n}=\int_{0}^{\infty} e^{-t} f^{\sim}(t) d t .
$$

If the $a_{n}=O\left((n !)^{\sigma}\right)$, for $\sigma<1$, then $f^{\sim}$ is entire, but not if $\sigma \geq 1$. In Borel's theory, the right side, whenever it makes sense, is defined as the Borel sum of the series on the left (if the series is absolutely convergent, the Borel sum coincides with the ordinary sum). For the Euler series, $f^{\sim}(w)=(1+w)^{-1}$ has a singularity at only $w=-1$ and so the Borel sum makes sense. We then have

$$
\sum_{n \geq 0}(-1)^{n} n ! z^{n} \sim \int_{0}^{\infty} \frac{e^{-w}}{1+w z} d w=: F(z)
$$

in the sense of Borel. The function $F(z)$ defined by the right side is analytic for $z=r e^{i \theta}$ with $|\theta|<\pi$ and is asymptotic to the formal power series on the left in the Poincaré sense:

$$
\left.\left|F(z)-\sum_{0 \leq m \leq n}(-1)^{m} m ! z^{m}\right|=O\left(|z|^{n+1}\right) \quad \text { uniformly in }|\theta| \leq \pi-\delta\right)
$$

for each $\delta>0$. Actually, the asymptotics holds in a sharper sense:

$$
\left.\left|F(z)-\sum_{0 \leq m \leq n}(-1)^{m} m ! z^{m}\right| \leq K_{\delta}(n+1) !|z|^{n+1} \text { for all }|\theta| \leq \pi-\delta, n \geq 0\right)
$$


where $K_{\delta}>0$ is a constant independent of $z$ and $n$; this is a refinement of the Poincare estimate that gives the dependence on $n$ also. It is referred to as strong asymptotics; one is allowed an additional $\sigma^{n}$ for the estimate of the remainder. It follows from a famous theorem of Watson that $F$ is uniquely determined by the factorial series as the only analytic function in the sector $|\theta|<\pi-\delta$ strongly asymptotic to it. With suitable modifications one can treat all of Euler's examples in an analogous manner.

The integral discussed above,

$$
\int_{0}^{\infty} \frac{e^{-w}}{1+z w} d w
$$

is the inverse Borel transform of $(1+z w)^{-1}$, which is the Borel transform of the factorial series $\sum_{n \geq 0}(-1)^{n} n ! z^{n}$. It is easy to see by a change of variables that it is the integral that Euler wrote. Indeed,

$$
\int_{0}^{\infty} \frac{e^{-w}}{1+x w} d w=\frac{1}{x} e^{1 / x} \int_{0}^{\infty} \frac{e^{-1 / t}}{t} d t \quad(w=1 / t-1 / x)
$$

Similarly, the series $g_{m p q}$ has the Borel transform

$$
\sum_{n \geq 0}(-1)^{n} x^{m+r q} \frac{p(p+q)(p+2 q) \ldots(p+(r-1) q)}{n !} w^{r}=x^{m} \frac{1}{\left(1+q x^{q} w\right)^{p / q}}
$$

so that the Borel sum of $g_{m p q}$ is the integral

$$
g_{m p q} \sim x^{m} \int_{0}^{\infty} \frac{e^{-w}}{\left(1+q x^{q} w\right)^{p / q}} d w .
$$

By the substitution $w=1 / q t^{q}-1 / q x^{q}$ this integral goes over to

$$
e^{\frac{1}{q x^{q}}} x^{m-p} \int_{0}^{x} e^{-1 / q t^{q}} t^{p-q-1} d t
$$

which is the integral obtained by Euler for this series. Since both the series $g_{m p q}$ and the associated integral depend on $x$ through $x^{q}$, it is better to work in the $\zeta$-plane where $\zeta=z^{q}$. Then the integral

$$
\int_{0}^{\infty} \frac{e^{-w}}{(1+q \zeta w)^{r}} d w \quad(r>0)
$$

is holomorphic on the cut plane with $|\arg (\zeta)|<\pi$, strongly asymptotic to

$$
\sum_{n \geq 0}(-1)^{n} \frac{r(r+1) \ldots(r+n-1)}{n !} \zeta^{n}
$$

on sectors $|\arg (\zeta)| \leq \pi-\delta$ for each $\delta>0$. I omit the details (see however [Bo, Ca $)$.

In my opinion, the understanding of these ultradivergent series as asymptotic expansions of solutions of differential equations having an integral representation, which is one of the many features of the work of Euler on the summation of such series, was a fantastic discovery. It would be more than a century before Poincaré $[\mathrm{P}$ and Emile Borel $[\mathrm{BO}$. rediscovered this theme as a part of the theory of linear meromorphic differential equations with irregular singularities and the asymptotics of their solutions. For these, unlike the Frobenius series when the singularity is regular, the formal solutions do not converge, although one can construct a full fundamental matrix of formal solutions. On any sector with vertex at one of the 
singularities, one can construct an analytic solution (germ of it actually), asymptotic to the formal matrix, in the sense that Poincaré introduced. Note that there are flat matrices of solutions in general and so the analytic solution is not unique, although summability methods allow one to pick the solutions in a canonical manner. In the modern versions of the theory these sheaves of flat functions, or rather their first cohomologies, form an obstruction to linking the formal theory with its analytic counterpart, and once they are taken into account, one can obtain a satisfactory theory [V1]. These summability methods have had great impact in many areas. This is however only one aspect of the theme that Euler started with his summation of the ultra divergent series of factorial type. The issues concerning the structure and numerical evaluations of the continued fractions of the Euler type, as well as their relation to the solutions of the differential equations, are still not fully resolved.

One last remark is appropriate before we wind up this brief discussion of divergent series and their summation. In modern analysis, especially in the theory of Fourier series and representation theory, another method of summation is widely used, the so-called smeared summation. Unlike most summation methods touched upon above, this method makes sense in all dimensions and has great flexibility. If $\left\{f_{n}(x)\right\}$ is a sequence of functions defined on $\mathbf{R}^{n}$ or the torus $T^{n}$ or, more generally, on a Lie group $G$, then

$$
\sum_{n} f_{n}(x)
$$

is summable in the weak sense if for all smooth functions with compact support $\varphi$, the sum

$$
\sum_{n} \int_{G} f_{n}(x) \varphi(x) d x=L(\varphi)
$$

is convergent; the limit $L$ is then a distribution in the sense of L. Schwartz. Very often $L$ is defined by a function $L(x)$, namely

$$
L(\varphi)=\int_{G} L(x) \varphi(x) d x,
$$

and then we say that

$$
\sum_{n} f_{n}(x)=L(x)
$$

in the sense of distributions. One is led to think of this as smeared summation because in many practical problems, the $f_{n}$ represent some physical quantities, and the actual value of $f_{n}(x)$ at a space time point is very difficult to determine exactly; only a space time average of $f_{n}$ around $x$ is measurable. One says that the measurement of $f_{n}(x)$ is smeared. This method was used by Harish-Chandra to define the characters of infinite dimensional irreducible unitary representations. Some of the main results of Fourier analysis on $G$ can be viewed as a formula expressing the Dirac delta at the identity as a linear combination of the characters of the irreducible unitary representations. The simplest of these is on the circle where the formula is

$$
\delta=\sum_{n=-\infty}^{\infty} e^{i n x} .
$$

Euler was interested in the sum on the right side. He proved, using his favorite Abel summation, that the right side is 0 except when $x \equiv 0 \bmod 2 \pi$. It is easy to 
see that if

$$
s_{N}(x)=\sum_{n=-N}^{N} e^{i n x}, \quad \sigma_{N}(x)=\frac{s_{0}(x)+s_{1}(x)+\ldots s_{N}(x)}{N+1},
$$

then

$$
\sigma_{N}(x) \rightarrow 0 \quad(N \rightarrow \infty)
$$

when $x \not \equiv 0 \bmod 2 \pi$ while

$$
\sigma_{N}(0)=N+1
$$

showing that the limit should be thought of in the smeared sense and is $\delta$. A proof is quite easy, using elementary Fourier analysis.

\section{Summation formula}

Throughout his life Euler was a tireless calculator, delighting in numerical calculations in almost all areas he worked in: astronomy, mechanics, infinite series, and so on. In the theory of infinite series he calculated the zeta values to a huge number of decimal places, using what we now call the Euler-Maclaurin summation formula. This was a favourite tool of his, and he used it in many ingenious ways to evaluate the results in many problems with great numerical accuracy. For instance he calculated to a huge number of decimal places what we now call Euler's constant, the summation value of the factorial series, the numerical value of $\pi=3.14 \ldots$, and so on.

The summation formula was read by Euler before the St. Petersburg Academy in 1734. Maclaurin discovered it independently around 1740. There was however no argument about priority; Euler just contented himself, in response to enquiries from Stirling, with the remark that the result is known and was read before the Academy in 1734. This was characteristic of him and his generous ways of accepting what others had done.

Let us recall the formula. If $f$ is a nice function, the summation formula asserts that

$$
\begin{aligned}
\frac{1}{2} f(0)+f(1)+f(2)+ & \cdots+f(N-1)+\frac{1}{2} f(N)=\int_{0}^{N} f(x) d x \\
& +\sum_{k=2}^{\infty} \frac{B_{k}}{(k) !}\left[f^{(k-1)}(N)-f^{(k-1)(0)}\right]
\end{aligned}
$$

where the $B_{k}$ are the Bernoulli numbers defined by

$$
\frac{1}{1-e^{-z}}=\frac{1}{z}+\frac{1}{2}+\sum_{k=2}^{\infty} \frac{B_{k}}{k !} z^{k-1} .
$$

One has, with the convention $B_{0}=1$,

$$
B_{1}=\frac{1}{2}, \quad B_{k}=0 \quad(k \geq 2, k \text { odd }), \quad B_{2}=\frac{1}{6}, \quad B_{4}=-\frac{1}{30}, \ldots
$$

Formally one wants to find $S(x)$ such that $f(x)=S(x)-S(x-1)$; if $D=d / d x$, then the above equation can be written as $\left(1-e^{-D}\right) S=f$ or $f=\left(1-e^{-D}\right)^{-1} f$, from which, expanding in powers of $D$, we get the result. It is possible to impose conditions on $f$ that make it clear that this is an asymptotic expansion: the error committed is at most the size of the last term taken into account. Euler knew of 
this because he always maintained that one has to stop when the terms of the series begin to diverge.

There is another way to understand this formula that links it with Fourier analysis and Poisson summation formula. I owe it (as well as the illustrative treatment of the Euler evaluation of the factorial series that follows as an application) to Pierre Deligne [D and I wish to describe it briefly.

Let $f$ be a function with compact support and of bounded variation and let its Fourier transform $\widehat{f}$ be defined by

$$
\widehat{f}(y)=\int f(x) e^{-2 \pi i x y} d x .
$$

Then we have (the Fourier integral analogue of Dirichlet's theorem for Fourier series)

$$
\frac{1}{2}[f(x+)+f(x-)]=\lim _{R \rightarrow \infty} \int_{-R}^{R} \widehat{f}(y) e^{-2 \pi i x y} d y .
$$

Let us now define the function on $\mathbf{R} / \mathbf{Z}$ (with the natural map $x \longmapsto \bar{x}$ )

$$
\bar{f}(\bar{x})=\sum_{n \in \mathbf{Z}} f(x+n) .
$$

Then $\bar{f}$ is of bounded variation and its Fourier coefficients are $\{\widehat{f}(m)\}_{m \in \mathbf{Z}}$. By Dirichlet's theorem we have

$$
\frac{1}{2}[\bar{f}(0+)+\bar{f}(0-)]=\sum_{m \in \mathbf{Z}} \widehat{f}(m),
$$

which is the Poisson summation formula in this context; the series on the right is in general not absolutely convergent, and one has to interpret it as a principal value, i.e., as $\lim _{M \rightarrow \infty} \sum_{m=-M}^{M} \widehat{f}(m)$. If $f$ is in addition continuous on $[0, N]$ and vanishes outside $[0, N]$, then

$$
\frac{1}{2}[\bar{f}(0+)+\bar{f}(0-)]=\frac{1}{2} f(0)+f(1)+\cdots+f(N-1)+\frac{1}{2} f(N)
$$

so that the above formula becomes

$$
\frac{1}{2} f(0)+f(1)+\cdots+f(N-1)+\frac{1}{2} f(N)=\int f(x) d x+\sum_{m \neq 0} \widehat{f}(m) .
$$

It is now a question of determining the asymptotic behaviour of the terms $\widehat{f}(m)$ that appear on the right side. This is controlled by the singularities of $f$ and its derivatives at 0 and $N$.

Assume now that $f$ is actually $C^{\infty}$ on the closed interval $[0, N]$ and vanishes outside $[0, N]$. Then, repeated integration by parts yields the asymptotics

$$
\widehat{f}(m) \sim \sum_{k=1}^{\infty} \frac{f^{(k-1)}(0)-f^{(k-1)}(N)}{(2 \pi i m)^{k}} \quad(m \rightarrow \infty) .
$$

So

$$
\sum_{m \neq 0} \widehat{f}(m) \sim \sum_{m \neq 0} \sum_{k=1}^{\infty} \frac{f^{(k-1)}(0)-f^{(k-1)}(N)}{(2 \pi i m)^{k}} \quad(m \rightarrow \infty) .
$$


We now interchange the order of summation of course. The terms for odd $k$ for $m$ and $-m$ cancel, while for even $k=2 r$ the summation over $m$ gives, using Euler's formulae (7) for $\zeta(2 r)$,

$$
(-1)^{r}(2 \pi)^{-2 r} 2 \zeta(2 r)=(-1)^{r}(2 \pi)^{-2 r}(-1)^{r-1} \frac{2^{2 r} B_{2 r}}{(2 r) !} \pi^{2 r}=-\frac{B_{2 r}}{(2 r) !} .
$$

This gives the summation formula. If one does not want to use the full asymptotic expansion for $\widehat{f}(m)$, one can stop at the term $k=2 r+2$ with a remainder term

$$
\frac{1}{(2 \pi i m)^{2 r+2}} \int_{0}^{N} f^{(2 r+2)}(x) e^{-2 \pi i x y} d x
$$

which is majorized by

$$
\left\|f^{(2 r+2)}\right\|_{1}(2 \pi m)^{-(2 r+2)}
$$

where $\|\cdot\|_{1}$ is the $L^{1}$-norm. We then see that the error in stopping at the $(2 r)^{\text {th }}$ term in the summation formula is

$$
\left|\frac{B_{2 r+2}}{(2 r+2) !}\right|\left(\left|f^{(2 r+1)}(N)-f^{(2 r+1)}(0)\right|+\left\|f^{(2 r+2)}\right\|_{1}\right) .
$$

If we assume (as Hardy does in his treatment [Ha]) that $f^{(2 r+2)}>0$ in $(0, N)$, we can replace the $L^{1}$-norm by

$$
\left|f^{(2 r+1)}(N)-f^{(2 r+1)}(0)\right|
$$

so that the error is

$$
2\left|\frac{B_{2 r+2}}{(2 r+2) !}\left(f^{(2 r+1)}(N)-f^{(2 r+1)}(0)\right)\right| .
$$

When one studies the convergence or summability of the Euler-Maclaurin series or is concerned with numerical evaluations, it is thus a question of balancing the growth of the derivatives of $f$ with that of the Bernoulli numbers. Euler was extremely skillful in such calculations. One should also remark that the expansion can equally be used to approximate the integral by the sum. Finally, the two features of the Euler formula, namely, the weights $1 / 2$ for the boundary terms and the Bernoulli numbers, enter Deligne's treatment for reasons different from the one's in Euler's original treatment: the boundary weights $1 / 2$ come from Dirichlet's theorem, and the Bernoulli numbers from the zeta values (see also [Ha, p. 330).

As an illustration let us use the summation formula to approximate

$$
\int_{0}^{1} h(t) d t \quad h(t)=\frac{e^{1-(1 / t)}}{t}
$$

by

$$
\delta\left[\frac{1}{2} h(0)+h(\delta)+h(2 \delta)+\cdots+h(9 \delta)+\frac{1}{2} h(1)\right] \quad\left(\delta=\frac{1}{10}\right),
$$

which Euler did in his first evaluation of the sum of the factorial series. To understand the surprising accuracy of this first evaluation we use the summation formula (applied to $f(t)=h(\delta t)(0 \leq t \leq 1 / \delta))$ to write the integral as a sum plus error terms. Now $h$ is flat at 0 and $h^{\prime}(1)=0$ and so the $B_{2}$ term is 0 . If we take the approximation through the term $B_{2} / 2$ !, the error committed is comparable to the first term omitted in the summation formula, namely to the size of the $B_{4}$ term. 
Now a simple calculation shows that $h^{(3)}(1)=4$. Hence the absolute value of the $B_{4}$ term is

$$
\frac{\left|B_{4}\right|}{4 !} \frac{1}{10^{4}}\left|h^{(3)}(1)\right|=\frac{1}{180 \times 10^{4}} \sim \frac{1}{10^{6}},
$$

which is the rough estimate of the error in Euler's approximation, giving at least a partial explanation as to why his first evaluation was so accurate.

\section{CONCLUding REMARKS}

I have not touched on many aspects of Euler's work of great current interest. For instance, in number theory he was indeed the great pioneer. He developed the foundations of the subject so that he could obtain the proofs of most of the assertions of Fermat, especially on problems involving the sums of two squares. He generalized the whole set up to one that asked what primes could be written in the form $x^{2}+N y^{2}$ for composite $N$, which led him to quadratic reciprocity and beyond; indeed, to survey his work from the modern point of view requires application of such sophisticated theories as class field theory. He discovered the product formula for the zeta function

$$
\zeta(s)=\prod_{p} \frac{1}{1-\frac{1}{p^{s}}}
$$

which he would write as

$$
1+\frac{1}{2^{s}}+\frac{1}{3^{s}}+\cdots=\frac{2^{s} \cdot 3^{s} \cdot 5^{s} \cdot 7^{s} \ldots}{\left(2^{s}-1\right)\left(3^{s}-1\right)\left(5^{s}-1\right)\left(7^{s}-1\right) \ldots}
$$

as well as the product formula for a few twisted series (with real characters mod 4, $6,8,12)$

$$
1+\frac{\chi(2)}{2^{s}}+\frac{\chi(3)}{3^{s}}+\cdots=\prod_{p} \frac{1}{1-\frac{\chi(p)}{p^{s}}} .
$$

From these he deduced, using their behaviour at $s=1$, for example that there are infinitely many primes in each residue class $p \equiv \pm 1(\bmod 4)$ and some classes mod 8. Ever since, such products over primes have been called Euler products. Almost a hundred years after Euler's discoveries Dirichlet would take up this theme and advance it spectacularly, associating Euler products to all characters, real or complex, for any modulus $N$, using their behaviour at $s=1$ to conclude that there are infinitely many primes in each residue class $\bmod N$ prime to $N$ for all moduli $N$. The further history of these ideas at the hands of modern masters such as Artin, Weil, Ramanujan, Deligne, Langlands, and a host of others is too well known to bear repetition here.

Inspired by Fagnano's work which came to his attention while he was at the Berlin Academy, Euler discovered the addition formula for elliptic integrals, which is the forerunner for the modern theory of elliptic curves and abelian varieties. It is of course impossible to go into detail about all of these. It is my hope that my discussion is enough to rekindle interest in Euler's work and to inspire younger people to look at his work with new eyes and focus.

\section{About the AUthor}

V. S. Varadarajan is a professor of mathematics at the University of California at Los Angeles. His research interests include representations of Lie and super 
Lie groups. His most recent books are Euler Through Time: A New Look at Old Themes and Super Symmetry for Mathematicians: An Introduction.

\section{REFERENCES}

[A] R. Apéry, Irrationalité de $\zeta(2)$ et $\zeta(3)$, Astérisque 61 (1979), 11-13.

[Bo] E. Borel, Leçons sur les Séries Divergentes, Éditions Jacques Gambay, 1988 (Reprinting of the original 1928 work).

[BL] E. J. Barbeau, and P. J. Leah, Euler's 1760 paper on divergent series, Historia Mathematica, 3(1976), 141-160. MR0504847 (58:21162a)

[BR] K. Ball and T. Rivoal, Irrationalité d'une infinité de valeurs de la fonction zeta aux entiers impairs, Invent. Math. 146(2001), no. 1, 193-207. MR1859021(2003a:11086)

[C] P. Cartier, Mathemagics (A Tribute to L. Euler and R. Feynman), Lecture Notes in Phys., vol. 550, Springer, Berlin, 2000, 6-67. MR1861978 (2003c:81002)

[Ca] B. Candelpergher, J. C. Nosmas, and F. Pham, Approche de la résurgence, Hermann, Paris, 1993. MR1250603 (95e:34005)

[D] P. Deligne, Letters (Personal communication).

[D1] P. Deligne, Lectures at UCLA, Spring 2005.

[DV] T. Digernes and V. S. Varadarajan, Notes on Euler's continued fractions (In preparation).

[E] L. Euler, Omnia Opera. All of Euler's papers in pure mathematics are in Series I of his Omnia Opera. For most of the questions discussed here, see I-8, I-14, I-15.

[E1] L. Euler, De seriebus divergentibus, Opera Omnia, I, 14, 585-617.

[Ha] G. H. Hardy, Divergent series, Oxford, 1973. MR0030620 (11:25a)

[Hi] Omar Hijab, Introduction to Calculus and Classical Analysis, Springer, 2007 (Second Edition). MR 1449395 (98e:26001)

[KZ] M. Kontsevich and D. Zagier, Periods, in 2001 and Beyond, 771-808, Springer, 2001. MR.1852188(2002i:11002)

[P] H. Poincaré, Sur les intégrales des équations linéaires, Acta Math. 8(1886), 295-344; Oeuvres, TI, Gauthier-Villars (1928), 290-332. MR.1554701

[R] T. Rivoal, Irrationalité d'au moins un des neuf nombres $\zeta(5), \zeta(7), \ldots, \zeta(21)$, Acta Arith. 103 (2002), no. 2, 157-167. MR.1904870 (2003b:11068)

[V] V. S. Varadarajan, Euler Through Time: A New Look at Old Themes, AMS, 2006. This is a partial source for almost all the matters discussed and the references for works mentioned in this article. MR2219954

[V1] V. S. Varadarajan, Linear meromorphic differential equations: a modern point of view. Bull. Amer. Math. Soc. (N.S.) 33 (1996), no. 1, 1-42. MR.1339809 (96h:34011)

Department of Mathematics, University of California, Los Angeles, Los Angeles, CALIFORnia 90095-1555

E-mail address: vsv@math.ucla.edu 